LA-UR-01-2890

Approved for public release; distribution is unlimited.

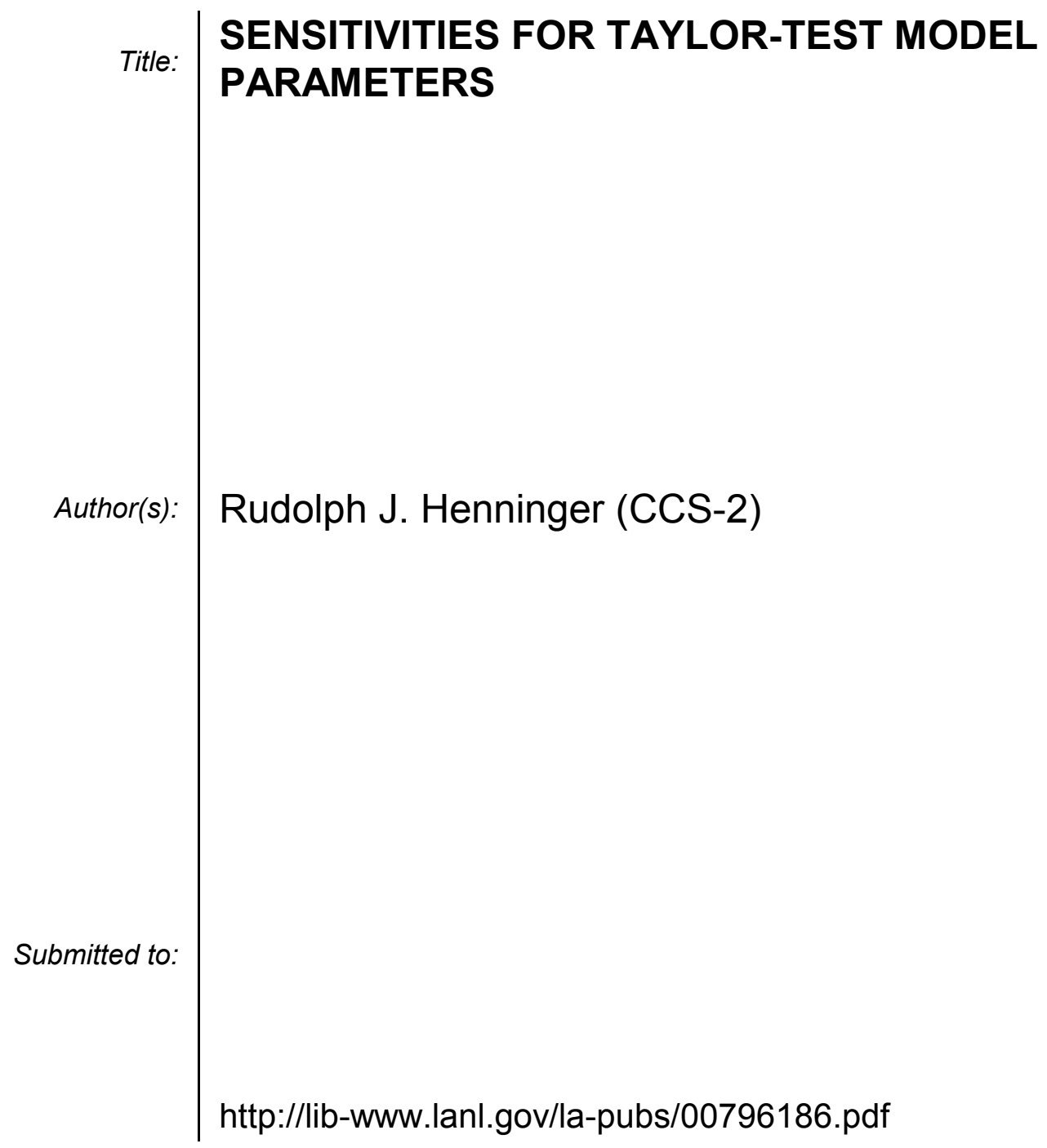

Los Alamos National Laboratory, an affirmative action/equal opportunity employer, is operated by the University of California for the U.S. Department of Energy under contract W-7405-ENG-36. By acceptance of this article, the publisher recognizes that the U.S. Government retains a nonexclusive, royaltyfree license to publish or reproduce the published form of this contribution, or to allow others to do so, for U.S. Government purposes. Los Alamos National Laboratory requests that the publisher identify this article as work performed under the auspices of the U.S. Department of Energy. Los Alamos National Laboratory strongly supports academic freedom and a researcher's right to publish; as an institution, however, the Laboratory does not endorse the viewpoint of a publication or guarantee its technical correctness. 


\title{
SENSITIVITIES FOR TAYLOR-TEST MODEL PARAMETERS
}

\author{
R. J. Henninger \\ Los Alamos National Laboratory, Los Alamos, New Mexico 87545
}

\begin{abstract}
The Taylor-Cylinder test has long been used to calibrate equation-of-state and materialstrength models. The process consists of impacting a cylinder of material on a rigid anvil and adjusting the model parameters to predict the resultant shape. Here we discuss the use of Automatic Differentiation (AD) of an Eulerian hydrodynamics model to provide sensitivities that are used as a gradient in the parameter fitting process. We apply AD in the forward and adjoint modes. For comparison, the gradient is also determined by finite differences. We find that adjoint methods provide the most efficient use of computational resources when there are 9 or more parameters.
\end{abstract}

\section{INTRODUCTION}

The purpose of this project has been to provide sensitivities of results from an Eulerian hydrodynamics computer code (hydrocode) (1) for use in design-optimization and uncertainty analyses. We began (2) by applying an equation-based sensitivity technique used successfully in the early eighties that was applied to reactor-safety thermalhydraulics problems $(3,4)$, which is called Differential Sensitivity Theory (DST) $(5,6)$. The methodology is as follows: the system of partial differential equations (the forward or physical PDEs) is assembled, and differentiated with respect to the model parameters of interest; the adjoint equations are then determined using the innerproduct rules of Hilbert spaces (5); and finally, the resulting adjoint PDEs are solved using straightforward numerical operators. The forwardvariable solutions when needed for the adjoint solutions are provided by the original computer code that solves the physical (or forward) problem. In the present hydrocode application, acceptable results were obtained for one-material, onedimensional problems. The DST results were then improved by means of "compatible" finite difference operators $(6,7)$. We have seen, however, that DST techniques do not produce accurate values for sensitivities to all of the parameters of interest and for problems with discontinuities such as a multi-material problem (8). To obtain accurate sensitivities for arbitrary numerical resolution a more code-based approach was then tried. Results for two-dimensional problems were obtained (9) by applying Automatic DIfferentiation of FORtran (ADIFOR, version 3.0) (10).

Here we present sensitivities for Taylor-cylinder impact test calculations. In what follows, we describe AD methods in the context of their use for a hydrocode. We then describe the Taylor-test calculations. This is followed by an examination of the results, accuracy, and computer run times for the ADIFOR-generated code. Finally, we outline our plans for future work.

\section{AUTOMATIC DIFFERENTIATION METHODS FOR A HYDROCODE}

Use of a hydrocode for experiment fitting purposes requires information about how some scalar result (or response, $R$ ) will change when some code parameter $(\alpha)$ is changed. This socalled sensitivity, $(\partial R / \partial \vec{\alpha})$, is the gradient (or Jacobian) that determines the search direction for obtaining an optimum response. Typically, the gradient is obtained by changing parameters one at a time to form a finite-difference (FD) derivative. This method requires $\mathrm{N}+1$ computer runs to determine sensitivities for $\mathrm{N}$ problem parameters.

Both code- and equation-based differential sensitivity methods can be implemented in either the forward or adjoint mode. By forward and 
adjoint, we mean the direction through the solution and in time and space in which the derivative values are obtained. The forward mode is more efficient for determining the sensitivity of many responses to one or a few parameters, while the adjoint mode is better suited for sensitivities of one or a few responses with respect to many parameters. Here we apply ADIFOR in both the forward and adjoint modes.

$\mathrm{AD}$ tools require several steps to get from the original code to an executable code that produces sensitivities. A precompiler first analyzes the code and modifies it to include code that calculates the derivatives of interest. In the forward mode the logic is straightforward. The required additional storage is simply added to the original code and the derivatives are calculated along with the forward solution. In the adjoint mode for a non-linear hydrocode the forward calculation must first be completed since the information from the forward calculation is needed in the adjoint or reverse calculation. Independent storage and/or recalculation can provide this information. The second step in the adjoint process is thus to determine and set up the required storage. For a large problem a technique called checkpointing is required. This technique consists of dumping the solution at checkpoints as the forward solution is generated. The complete forward solution is stored from the final checkpoint to the final time of the forward calculation. One then calculates the adjoint solution backward from the final state to the last checkpoint. The forward solution is then calculated and stored from the second-to-the-last to the last checkpoint. The adjoint solution is then generated from the last to the second-to-last checkpoint. This process is repeated until the initial time of the forward calculation is reached and the sensitivities are complete.

\section{TAYLOR-CYLINDER IMPACT CALCULATIONS}

In this subsection we obtain sensitivities for a copper Taylor-cylinder impact test (11). In the experiment, a $0.762-\mathrm{cm}$ diameter $2.54-\mathrm{cm}$ long copper cylinder at 146 or $190 \mathrm{~m} / \mathrm{s}$ strikes a rigid anvil. We use a Mie-Gruneisen equation of state (EOS) (12) and the Steinberg-Cochran-Guinan strength model (13) to represent the copper. As implemented in the code, the pressure $p$ and yield strength $Y$ are given by

$$
\begin{aligned}
& p=p_{H}+\Gamma \rho_{0}\left[e-\frac{1}{2} p_{H}\left(\frac{1}{\rho_{0}}-\frac{1}{\rho}\right)\right] \\
& p_{H}=c_{0}^{2} \rho\left(\frac{\rho}{\rho_{0}}-1\right) /\left[1-\left(\frac{\rho}{\rho_{0}}-1\right)(s-1)^{2}\right] \\
& Y=Y_{0}\left[1+\alpha\left(\varepsilon_{0}^{p}+\varepsilon_{0}\right)^{\beta}\right][1+\gamma p] e^{-\left(\delta e /\left(e_{m}-e\right)\right)}
\end{aligned}
$$

Where $\rho$ is the density, $e$ is the internal energy, $\varepsilon_{0}$ is the plastic strain, and $\gamma$ is the pressure-hardening coefficient. The remaining model parameters are defined in Table 1. We intend to use the sensitivities to find the model parameters that best match the final shape of the copper cylinder. The response is therefore chosen to be

$R=C \sum_{k}\left(r_{\text {calc }, k}-r_{\text {exp }, k}\right)^{2}$.

Where $C$ is a constant, $r_{\exp , k}$ is the $\mathrm{k}^{\text {th }}$ experimental final radius, and $r_{c a l c, k}$ is the $\mathrm{k}^{\text {th }}$ calculated final radius. Each of the $\mathrm{k}$ radii is at the same axial location. The sensitivities of this response to the initial conditions and the EOS and strength parameters along with their definitions and nominal values are given in Table 1 . If one normalizes by multiplying each sensitivity by its nominal parameter value, the parameters can be ranked as to their importance. We see that the nominal EOS density is the most important followed by the cylinder initial velocity and the strain hardening exponent. The table also compares the AD sensitivities to the FD sensitivities determined with a fractional parameter perturbation size of $10^{-7}$. The agreement is only fair for several of the parameters. We compare the AD and FD results by looking at the time-dependence of the sensitivities. Two of the important sensitivities are shown in Fig. 1. The agreement is quite good. Examination of the $c_{0}$ sensitivity that did not agree well shows that it agreed well to approximately $30 \mu \mathrm{s}$; then diverged as is shown in Fig. 2. As seen in the figure a smaller perturbation fraction $\left(10^{-8}\right)$ agrees better at late time. Close examination of all of the FD sensitivities shows that large fractions lose accuracy and small fractions display truncation errors. The comparisons are similar for the $146 \mathrm{~m} / \mathrm{s}$ case. The FD sensitivities for this problem are thus useful for rough confirmation only. The accuracy required when using the sensitivities as a gradient for an optimization process or uncertainty analysis is yet to be determined. 
TABLE 1. Comparison of the Taylor-cylinder test sensitivities for a $190 \mathrm{~m} / \mathrm{s} \mathrm{impact.}$

\begin{tabular}{|c|c|c|c|c|}
\hline Parameter description and value & $\begin{array}{c}\text { AD forward \& } \\
\text { adjoint } \\
\text { sensitivity }\end{array}$ & $\begin{array}{l}\text { AD value } \\
\text { normalized }\end{array}$ & Rank & $\begin{array}{c}\text { FD sensitivity } \\
(1.0 \mathrm{E}-7)\end{array}$ \\
\hline Initial density, $\rho(t=0)\left(8.93 \mathrm{~g} / \mathrm{cm}^{3}\right)$ & 0.0038838 & $3.47 \mathrm{E}-02$ & 4 & 0.058777 \\
\hline Initial velocity, $u z(t=0)(0.0190 \mathrm{~cm} / \mu \mathrm{s})$ & 3.7952 & $5.54 \mathrm{E}-02$ & 2 & 4.2063 \\
\hline Initial internal energy, $e(t=0)\left(0 \mathrm{Mbar}-\mathrm{cm}^{3} / \mathrm{g}\right)$ & 0.78148 & 0.00 & - & 0.99517 \\
\hline Initial stress deviator, $\operatorname{szz}(t=0)(0$ Mbar $)$ & -0.22942 & 0.00 & - & -0.029852 \\
\hline Shock velocity constant, $s(1.489)$ & $-4.6204 \mathrm{e}-05$ & $6.88 \mathrm{E}-05$ & 9 & $-4.4867 e-05$ \\
\hline Sound speed, $c_{0}(0.3940 \mathrm{~cm} / \mu \mathrm{s})$ & 0.022017 & $8.67 \mathrm{E}-03$ & 7 & -0.0062747 \\
\hline Nominal EOS density, $\rho_{0}\left(8.93 \mathrm{~g} / \mathrm{cm}^{3}\right)$ & -0.0078693 & $7.03 \mathrm{E}-02$ & 1 & -0.062284 \\
\hline Gruneisen ratio, $\Gamma(2.002)$ & -0.00013854 & $2.77 \mathrm{E}-04$ & 8 & -0.00013871 \\
\hline Linear artificial viscosity constant $(0.2)$ & $-7.3006 \mathrm{e}-05$ & $1.46 \mathrm{E}-05$ & 11 & -0.00010793 \\
\hline Quadratic artificial viscosity constant (2.0) & $-1.1642 \mathrm{e}-06$ & $2.33 \mathrm{E}-06$ & 13 & $-1.1569 \mathrm{e}-06$ \\
\hline Strain hardening constant, $\alpha$ (36) & 0.00038914 & $1.40 \mathrm{E}-02$ & 6 & 0.00039092 \\
\hline Pre-strain for wrought materials, $\varepsilon_{0}^{p}(0)$ & 0.029650 & 0.00 & - & 0.029319 \\
\hline Strain hardening exponent, $\beta(0.45)$ & 0.095916 & $4.32 \mathrm{E}-02$ & 3 & 0.11580 \\
\hline Thermal softening coefficient, $\delta$ (0.001) & 0.0016448 & $1.64 \mathrm{E}-06$ & 14 & -0.0016989 \\
\hline Melt energy, $e_{m}\left(0.0571 \mathrm{Mbar}-\mathrm{cm}^{3} / \mathrm{g}\right)$ & 0.00030827 & $1.76 \mathrm{E}-05$ & 10 & 0.00032079 \\
\hline Nominal yield stress, $Y 0$ (0.0012 Mbar) & 27.454 & $3.29 \mathrm{E}-02$ & 5 & 27.572 \\
\hline Shear modulus (0.477 Mbar) & $-2.7090 \mathrm{e}-05$ & $1.29 \mathrm{E}-05$ & 12 & -0.0017957 \\
\hline
\end{tabular}

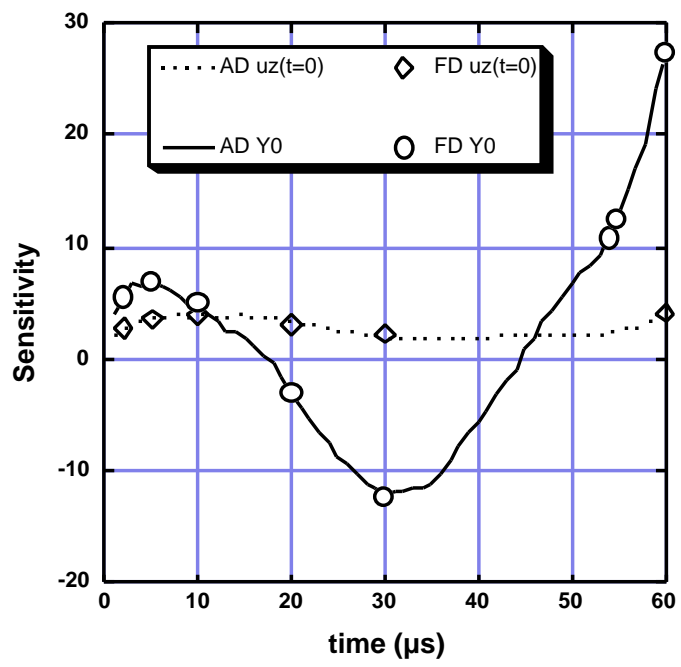

FIGURE 1. Comparison of AD- and FD- produced nominalyield-stress and initial-velocity sensitivities for a $190 \mathrm{~m} / \mathrm{s}$ impact.

An important issue when choosing whether to use a forward or adjoint method to determine sensitivities is the amount of computer time that each method requires. As mentioned above, the number of parameters versus the number of responses plays a key role in this decision. For this

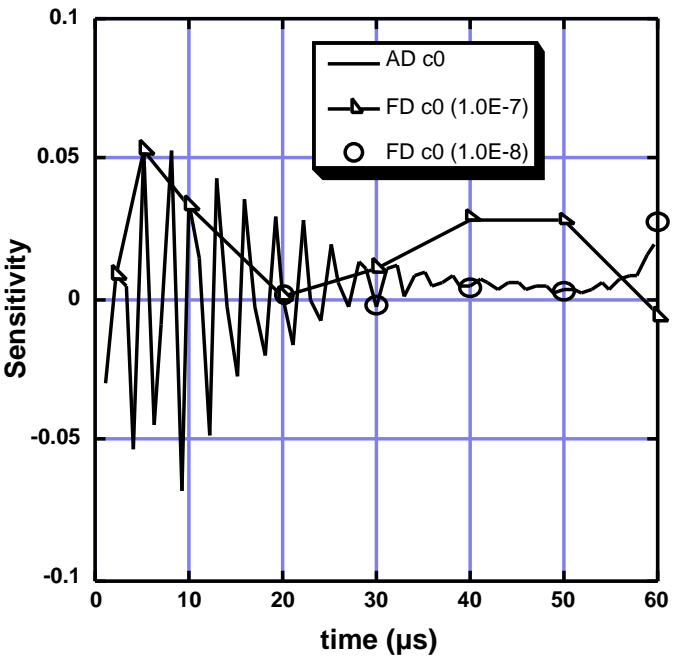

FIGURE 2. Comparison of AD- and FD- produced sound-speed sensitivities for a $190 \mathrm{~m} / \mathrm{s}$ impact. The FDs were determined with two fractional perturbations $\left(10^{-7}\right.$ and $\left.10^{-8}\right)$.

problem we have chosen only one response. The run times for the various methods used to obtain the results in Table 1 are listed in Table 2.

We see that ADIFOR-adjoint uses the least CPU time. For a problem like this one with only one response additional or fewer parameters will not 
significantly change the ADIFOR-adjoint run time. ADIFOR-forward and FD runtimes scale linearly with the number of parameters. One could determine the sensitivities for approximately 9 parameters in the adjoint runtime using the ADIFOR-forward or FD methods. This becomes the break-even number for the ADIFOR-adjoint method. In other words, for 9 or more problem parameters the adjoint method will make best use of computational resources. Here we used uniformly spaced checkpoints (every $1 \mu \mathrm{s}$ for the $60 \mu$ s calculation). This may not be the optimal balance of storage versus recalculation (14). As we gain experience with these methods, minimization of computational time will be better understood and pursued.

TABLE 2. SGI Origin 2000 CPU times for Taylor-test calculation with 17 parameters.

\begin{tabular}{llll}
$\begin{array}{l}\text { Single } \\
\text { Forward } \\
\text { (CPU sec.) }\end{array}$ & $\begin{array}{l}\text { ADIFOR } \\
\text { Adjoint } \\
\text { (CPU sec.) }\end{array}$ & $\begin{array}{l}\text { ADIFOR } \\
\text { Forward } \\
\text { (CPU sec. })\end{array}$ & $\begin{array}{l}\text { Finite } \\
\text { Difference } \\
\text { (CPU sec.) }\end{array}$ \\
\hline 1935 & 21141 & 38986 & 37316
\end{tabular}

\section{SUMMARY AND FUTURE WORK}

We have applied the automatic differentiation tool ADIFOR (version 3.0) to the MESA2D hydrocode (a Fortran77 code) and have obtained accurate sensitivities for a Taylor-cylinder impact problem in both the forward and adjoint modes. We have determined for problems of this size and duration that the adjoint method is most efficient when there are 9 or more problem parameters. We will apply this capability to experimental-data assimilation and result-uncertainty analysis with this code. We will then extend the capability to parallel codes written in languages other than Fortan77.

\section{ACKNOWLEDGEMENTS}

Work performed under the auspices of the U. S. Department of Energy at the Los Alamos National Laboratory under Contract W-7405-ENG-36.

\section{REFERENCES}

1. D. J. Cagliostro, D. A. Mandell, L. A. Schwalbe, T. F. Adams, and E. J. Chapyak, "MESA 3-D Calculations of Armor Penetration by Projectiles with Combined
Obliquity and Yaw," Int. J. Impact Engineering, 10, (1990).

2. P. J. Maudlin, R. J. Henninger, and E. N. Harstad, "Application of Differential Sensitivity Theory to Continuum Mechanics," Proc. ASME Winter Annual Meeting, 93, New Orleans, Louisiana (November 28December 3, 1993).

3. P. J. Maudlin, C. V. Parks and C. F. Weber, "Thermal-Hydraulic Differential Sensitivity Theory," ASME paper No. 80-WA/HT-56, Proc. ASME Annual Winter Conference (1980).

4. C. V. Parks and P. J. Maudlin, "Application of Differential Sensitivity Theory to a Neutronic/Thermal Hydraulic Reactor Safety Code," Nucl. Technol., 54, 38 (1981).

5. D. G. Cacuci, C. F. Weber, E. M. Oblow and J. H. Marable, "Sensitivity Theory for General Systems of Nonlinear Equations," Nucl. Sci. Eng., 75, 88 (1980).

6. R. Henninger, P. Maudlin, and M. L. Rightley, "Accuracy of Differential Sensitivity for OneDimensional Shock Problems," LA-UR-97-596, 1997

APS Shock Compression of Condensed Matter Conference, pages 187-190, Amherst, MA (27 July -1 August 1997).

7. M. Shashkov, Conservative Finite Difference Methods on General Grids, CRC Press, Boca Raton (1996).

8. R. J. Henninger, P. J. Maudlin, and E. N. Harstad, "Differential Sensitivity Theory Applied to the MESA2D Code for Multi-Material Problems," Proceedings of the APS Meeting on Shock Compression of Condensed Matter, pages 283-286, Seattle, WA, (August 1995).

9. Rudy Henninger, Alan Carle, and Paul Maudlin, "Hydrocode Sensitivities by Means of Automatic Differentiation," Third International Symposium on Sensitivity Analysis of Model Output (to be published), Madrid, Spain (18-20 June 2001).

10. - "ADIFOR 3.0 Overview", Rice University Technical Report CAAM-TR-00-02 (February 2000).

11. Marc A. Meyers, Dynamic Behavior of Materials, page 82, John Wiley \& Sons, Inc., New York (1994).

12. F. H. Harlow and A. A. Amsden, "Fluid Dynamics," Los Alamos Scientific Laboratory Report LA-4700, 1971.

13. D. J. Steinberg, S. G. Cochran, and M. W. Guinan, "A Constitutive Model for Metals at High Strain Rate," J. Appl. Phys. 51 (3), page 1498 (1980).

14. Andreas Griewank, Evaluating Derivatives: Principles of Algorithmic Differentiation, SIAM Frontiers in Applied Mathematics, page 319, Philadelphia (2000). 